\title{
Work attitude in high school students in times of pandemic
}

Actitud laboral en estudiantes secundarios en tiempos de pandemia

\section{Vega Chumpitaz María Aurora}

Universidad César Vallejo, https://orcid.org/0000-0003-2123-2495, vangulo@regioncallao.gob.pe

\section{Revista Iberoamericana de la Educación Vol - Especial 12021 \\ e-ISSN: 2737-632x}

\begin{abstract}
The problem of pandemic, the overcrowding due to COVID 19, has greatly changed the attitude of many families causing Young people to have to Word al an early age, in other cases they stop studying to assume roles that do not correspond to them playing the role of mother or father, to motivate themselves, with an entrepreneurial spirit developing it in their environment, that is why the teacher must be trained to raise awareness in all the technological transformations that accompany their pedagogical and socio- emotional work in student care.

The objective of the study was to determine the level of work attitude in secondary school students in times of pandemic, with a quantitative approach and simple descriptive design, and the survey technique was used and as an instrument the questionnaire was applied to a probabilistic sample of $151 \mathrm{fifth}$ grade high school students, concluding: Fifth grade high school students from the educational institution Dionisio Manco Campos have a positive but not significant work attitude.
\end{abstract}

Key words: work attitude, motivation, students 
Resumen: El problema de la pandemia, el hacinamiento debido al COVID 19, ha cambiado mucho la actitud de muchas familias haciendo que los jóvenes tengan que trabajar a temprana edad, en otros casos dejan de estudiar para asumir roles que no les corresponde haciendo el papel de madre o padre, para llevar el sustento a sus hogares, creando nuevas formas de motivarse, con un espíritu emprendedor desarrollándolo en su entorno, es por eso que el docente debe estar capacitado para sensibilizar en todas las transformaciones tecnológicas que acompañan en su labor pedagógica y socioemocional en atención del estudiante.

Este estudio tuvo como objetivo determinar el nivel de actitud laboral en los estudiantes secundario en tiempos de pandemia, con un enfoque cuantitativo y diseño descriptivo simple, y se utilizó la técnica de la encuesta y como instrumento el cuestionario que se aplicó a una muestra probabilística de 151 estudiantes de quinto grado de secundaria, llegando a concluir: Los estudiantes del quinto grado de secundaria de la institución educativa Dionisio Manco Campos presentan una actitud laboral positiva pero no significativa.

Palabras clave: actitud laboral, motivación, estudiantes

\section{INTRODUCTION}

A fines de diciembre del 2019, se informó sobre muchos casos de fuerte gripe, cuyo origen seria en Wuhan, una provincia de China, asi lo expresa, en su investigación Sheposh (2019) inicialmente denominó sars - CoV 2 Covid-19 (OMS 2020) se extendió de manera rápida en todo los países, con casos confirmados de muerte, mucha desolación familiar en el Perú se declara el derecho a la protección y prevención (D.S.008-2020 S.A.) Logró desafiar los sistemas de salud pública afirman Urzúa ét al (2020) afectó la estructura socioeconómica mundial y nacional, se constituye en un reto para muchos 
jóvenes estudiantes, según su trabajo publicado por Vicentini (2020) agrega, con el cierre de mercados e instituciones educativas, generado por estas nuevas medidas y el uso de la tecnología.

Ryder (2020) señalo con el afán de una adecuada formación, se ha visto afectado emocionalmente, según Organización mundial del trabajo el 65\% de estudiantes ha presentado rechazo a las clases virtuales siendo un $9 \%$ ha abandonado las escuelas, lo describen Avendaño, Luna y Quintero (2020).este golpe a provocado que mentalmente se pierda a un futuro profesional, tanto en su formación como en su educación, se debe permitir el desarrollo de la competencia para hacer frente a las exigencias del sector productivo para los momentos después de la pandemia.

De acuerdo con Velasco (2020), se puede apreciar el desarrollo de actitudes laborales positivas, el emprendimiento implica una idea de mejora para la transformación de la sociedad, Sorbara et al (2021) plantean muchos jóvenes están dispuestos a trabajar, apoyar a sus familias, muchos padres han perdido sus trabajos, o perdido la vida, otros por embarazo precoz, están asumiendo roles que no le corresponden. Desde el punto de vista, Lewis (2019) mencionó la familia cumple un papel importantísimo en la sociedad fomentando valores, afectivos, de convivencia, de alimentación de cuidados entre otros. Se mide el grado de estudios profesionales y muchos de ellos no cuentan con la experiencia, la edad, ni con el título que avale su desempeño, asi lo describe Suarez (2020 p.38) En América Latina y el Caribe las pymes son consideradas como punto estratégico de la económica también se ha calificado como la generación de empleo en la pequeña y mediana empresa (CEPAL 2020); en el Perú la simplificación de documentos ha motivado que las ONG ayuden a formar sus ideas de emprendimiento, en puesto de negocio, la intervención oportuna de la SUNAT (2020), ha dado facilidades para la formalización para la micro y pequeñas empresas. 
La aparición en los últimos años de la Comisión Europea (2012) publica una nueva visión del docente aplicando nuevas competencias laborales las cuales se pueden insertar y socializar con el estudiante; así, en Colombia desde el 2006 se aprobó la ley donde el docente debe promover el espíritu emprendedor en todos los niveles hasta superior con la finalidad de fomentar en el estudiante la creación de empresas desde sus inicios hasta la formalización. (Comisión Europea, 2020)

En la opinión de Melo y Silva (2021) señalan la educación peruana y el sistema productivo, logró convalidar actitudes laborales requeridas por el sector empresarial, muchos estudiantes tienen que trasladarse de la zona rural a la zona urbana por falta de mercado, además Sánchez et al (2018) consideran sobre un cambio estructural de la economía, demanda formación en transferibilidad, versatilidad, conocimiento científico tecnológico.

Por otra Euler (2018 p.3) considera polivalencia, la problemática para los recién egresados de la educación básica regular, enfrenta a un mercado laboral saturado, se sustenta en el informe técnico del INEI, donde concluye que, la educación secundaria en el 2019, 53,2\% solo estudia, el 7,2\% solo trabaja, y el $23,5 \%$ solo estudia y trabaja $11.3 \%$ no estudia ni trabaja (Instituto Nacional de Estadística, 2020)

Asi mismo Heredia (2020) destaca en el Perú, la educación tiene un rol esencial de todo ser humano, con una responsabilidad de brindar ambientes seguros a todos los estudiantes, donde puedan socializar, aprender hallar soporte emocional Cardona et al (2008) agregan las competencias laborales y García (2020) sugiere darles la oportunidad de desarrollar competencias laborales que le permitan desenvolverse a lo largo de la vida; Anaya et al (2021) agregan el cierre de las instituciones educativa, trae consecuencias negativas en los estudiantes en relación a sus aprendizajes, desarrollo 
socioemocional, inteligencia emocional el logro de competencia laborales descrito por Cáceres et al (2020)

La emblemática Institución Educativa Dionisio Manco Campos, al sur de Lima, con 59 años de fundación, donde empodero a muchos jóvenes, con ideas futuristas de emprendimiento quienes en la actualidad ocupan cargos públicos muy importantes, como profesionales, a nivel local, nacionales e internacionales, cuenta con 247 estudiantes, de quinto grado, culminan la secundaria cada año, Fuentes (2020) destaca en tal sentido, es necesario que desarrollen competencias laborales que fortalezca su actitud empresarial mientras dure la emergencia sanitaria Minedu (2021)

Además, Mala es un distrito acogedor con mucho potencial en recursos naturales, minería, flora y fauna bañada por un hermoso mar donde los turistas visitan todo el año donde aún se conserva las tradiciones de antaño como sus platos típicos a base de pescado, mariscos y otros, fiestas costumbristas, zonas arqueológicas. (MuniMala 2019)

Desde el punto de vista Curulies y Schamme (2021) proponen es necesario un empoderamiento tomarlo en cuenta, recursos de la zona en especial la capacitación dirigida a los docentes del área educación para el trabajo e implementar una tecnología adecuada, proponen Pinto y Plaza (2021) el fortalecimiento las capacidades de las actitudes empresariales, de creatividad, asertividad, trabajo cooperativo, la capacidad de resolver un problema, capacidad en emprender, de indagar, donde podamos visualizar las potencialidades del entorno

Además, hay que tener en cuenta no existe una estrategia con tácticas diferenciadas de establecer alianzas con el sector manufacturero, desde la posición de Dubs (2021) Para el desarrollo de competencias laborales implica responder demandas específicas y abrir espacios de observación, agregan Hernández et al (2021) la práctica que permitan a los estudiantes explorar 
intereses profesionales, productivos, ejercitar las competencias laborales aprendidas.

Ante el problema descrito, se formula la siguiente interrogante: ¿Cuál es el nivel de actitud laboral en los estudiantes del quinto grado de secundaria?; así también se formuló el objetivo: determinar el nivel de actitud laboral en los estudiantes del quinto grado de secundaria.

El estudio se justifica porque permitió incrementar y actualizar conocimientos teórico científicos acerca de la actitud laboral en los estudiantes, sustentado por autores nacionales e internacionales actualizadas acorde a los tiempos de pandemia; de la misma manera, los resultados del estudio permitirán a las autoridades educativas, poder tomar decisiones acerca de la problemática, en la búsqueda de una formación integral de aquellos estudiantes que egresan el nivel secundario.

También, porque en respuesta a un enfoque cuantitativo, se realizó el recojo de datos de manera numérica, y un posterior análisis estadístico en la comprobación de los resultados, así como poder implementar espacios curriculares desatendidos acorde a enfoques y teorías sobre emprendimiento y actitud laboral.

\section{MATERIALS AND METHODS}

La investigación está orientado a un enfoque cuantitativo, que de acuerdo a Hernández, Fernández y Baptista (2014), es de carácter numérico, donde se realiza un análisis estadístico con los resultados obtenidos de la aplicación de instrumentos válidos y confiables.

El tipo de investigación fue descriptivo, que de acuerdo a Carrasco (2019), permite el registro del proceso de enseñanza y aprendizaje al docente investigado, proporciona estudios básicos para descubrir problemas de 
fenómenos educativos, así como diagnósticos situacionales del fenómeno educativo.

Asimismo, la población, para Hernández, Fernández y Baptista (2014), es el conjunto de todos los casos que concuerdan con una serie de especificaciones. La población estará conformada por 247 estudiantes del quinto grado de secundaria de la Institución educativa Dionisio Manco Campos de Mala, según se detalla a continuación:

Tabla 1

Distribución de la población

\begin{tabular}{ll}
\hline Sección & Total \\
\hline A & 31 \\
B & 32 \\
C & 30 \\
D & 32 \\
E & 30 \\
F & 31 \\
G & 31 \\
H & 30 \\
\hline TOTAL & 247 \\
\hline
\end{tabular}

Dato. Nóminas de matrícula

La muestra según Hernández, Fernández y Baptista (2014), es una porción representativa de la población, con los mismos rasgos y características, y para efectos del estudio fue probabilística; y se seleccionará mediante la fórmula Atkin y Colton (1995) 


$$
m=\frac{N \cdot Z^{2} \cdot p \cdot q}{(N-1) e^{2}+Z^{2} \cdot p \cdot q}
$$

Donde Z: Nivel de confianza $(1,96)$

$\mathrm{P}:$ eventos positivos $(0,5)$

Q: Eventos negativos $(0,5)$

N: Población 247

E: Margen de error $(0,05)$

\section{El muestreo}

El muestreo fue probabilístico, aleatorio y estratificado proporcional, que comprende todos los miembros de la población tuvieron la misma oportunidad de ser elegido como miembro de la muestra

$$
\begin{aligned}
& m=\frac{(247)(1,96)^{2}(0.5)(0.5)}{(247-1)(0,05)^{2}+(1,96)^{2}(0.5)(0.5)}=\frac{(247)(0,9694)}{0,615+(0,9604} \\
& m=\frac{237,2188}{1,5754}=150,57 \\
& m=151
\end{aligned}
$$

Se distribuyó la muestra entre las aulas, según el coeficiente de estratificación proporcional:

$$
f h=\frac{n}{N}=\frac{151}{247}=0,611
$$


Tabla 2

Distribución de la muestra

\begin{tabular}{cccc}
\hline Sección & & $F h$ & Total \\
\hline A & 31 & 0,611 & 19 \\
B & 32 & 0,611 & 20 \\
C & 30 & 0,611 & 18 \\
D & 32 & 0,611 & 20 \\
E & 30 & 0,611 & 18 \\
F & 31 & 0,611 & 19 \\
G & 31 & 0,611 & 19 \\
H & 30 & 0,611 & 18 \\
\hline Total & 247 & & 151 \\
\hline
\end{tabular}

La técnica utilizada fue la encuesta, de acuerdo a Tamayo (2012), es el procedimiento que recoge información directa o indirecta con un formulario de preguntas

El instrumento fue un cuestionario, que de acuerdo a Naupas ét al. (2013), Consiste en la formulación de un conjunto sistemático de preguntas que están relacionadas a hipótesis de investigación y por ende a variables e indicadores de investigación, se realizó la confiabilidad de los instrumentos, que según Hidalgo (2006) es la representación del grado de similitud de las respuestas que son observadas entre lo que la investigadora quiere investigar y el integrante de la muestra, y para efectos de la investigación. 


\section{RESULTS}

Luego de la aplicación del cuestionario, se obtuvo el siguiente resultado:

Tabla 3

Distribución de niveles de la actitud laboral en estudiantes de quinto de secundaria

\begin{tabular}{lll}
\hline Niveles & Frecuencia & Porcentaje \\
\hline Bajo & 54 & 35.7 \\
Medio & 75 & 49.7 \\
Alto & 22 & 14.6 \\
\hline Total & 151 & 100.0 \\
\hline
\end{tabular}

Actitud laboral

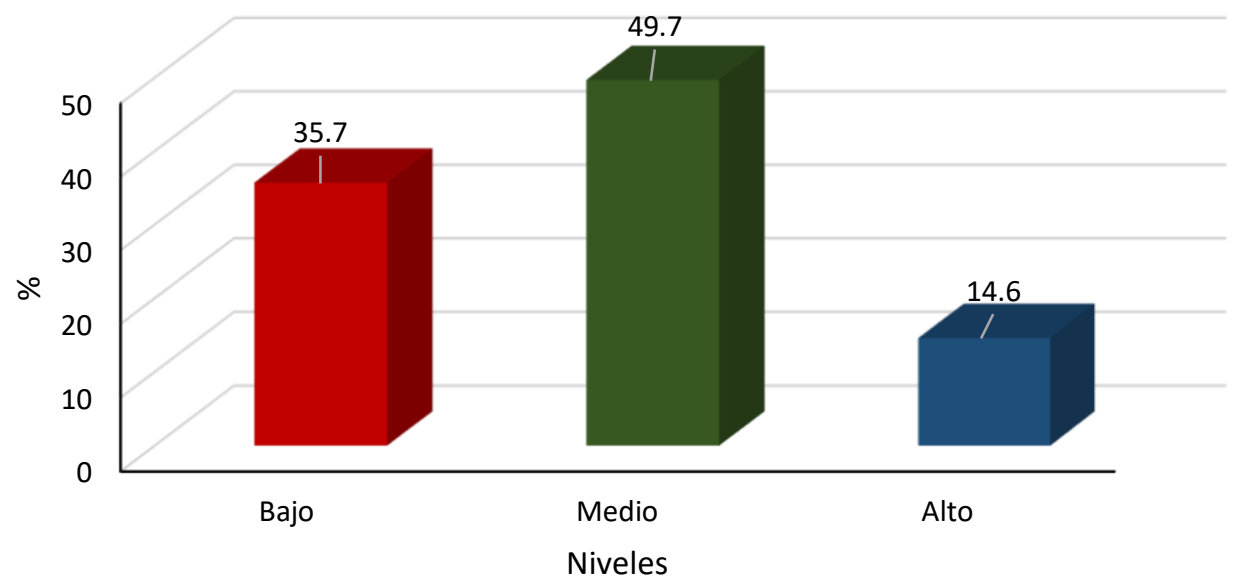

Figura 1. Niveles de la actitud laboral en estudiantes de quinto de secundaria 
En los resultados obtenidos, según la tabla 3 y figura 1, se observa que, los estudiantes del quinto grado de secundaria, presentan un $49,7 \%$ en el nivel medio, el $35,7 \%$ en el nivel bajo y el $14,6 \%$ un nivel alto; lo que indica que la mayoría de los estudiantes presentan entre un nivel bajo y medio en la actitud laboral.

\section{DISCUSSION}

De los resultados obtenidos se afirma que, los estudiantes del quinto grado de secundaria de la institución educativa Dionisio Manco Campos presentan niveles bajos y medio de actitud laboral, por lo que se puede afirmar que no presentan una actitud positiva significativa.

\section{CONCLUSIONS}

Se comprobó que el problema del estudiante egresado, frente a la actitud laboral, debe fortalecerse por parte de los aliados estratégicos empresariales, el Minedu, directores de colegios, docentes y padres de familia, atender el deseo de los jóvenes por su empoderamiento al trabajo a pesar de no tener edad ni documentos, de esta manera, puedan insertarse a trabajo de medio tiempo digno sin explotación.

La disposición de los docentes, es una fortaleza para los jóvenes porque ellos están dispuestos apoyar pero necesitan conocer más del lugar porque muchos son contratados, y desconocen las costumbres locales. 


\section{REFERENCES}

Anaya T., Montalvo J., Calderón I., y Arispe C. (2021). Escuelas rurales en el Perú: factores que acentúan las brechas digitales en tiempos de pandemia (COVID- 19) y recomendaciones para reducirlas.

Educación, 30(58),

11-33. https://doi.org/10.18800/educacion.202101.001

Atkin H. y Colton R. (1995). Métodos estadísticos. México: Prentice Hall.

Avendaño W., Luna H. y Quintero L. (2020). La política colombiana de emprendimiento en educación y su impacto en el acceso al empleo de jóvenes. El Ágora USB, 20(2), 158-171

Cáceres L., García R. y García , O.(2020) El manejo de la inteligencia emocional en los estudiantes de secundaria. Un estudio exploratorio en una telesecundaria en México. Conrado [online]. 2020, vol.16, n.74 [citado 2021-09-27], pp.312-324. Disponible en: <http://scielo.sld.cu/scielo.php?script=sci_arttext\&pid=S199086442020000300312\&lng=es\&nrm=iso >. Epub 02-Jun-2020. ISSN 25197320 .

Carrasco, S. (2019). Metodología de investigación científica. Pautas metodológicas para diseñar y elaborar el proyecto de investigación. Lima: Editorial San Marcos.

Cardona M, Vera L, Tabares J. (2008) Las dimensiones del emprendimiento empresarial la experiencia de los programas cultura e y fondo empresarial en Medellín grupo de estudios sectorial y territorial ESYT, departamento de economía escuela de administración universidad EAFIT ISSN1692 0694 
CEPAL (2020). La educación en tiempos de la pandemia de COVID-19. Santiago: CEPAL.

Comisión Europea (2020). Plan de acción sobre emprendimiento 2020. Bruselas: C.E

Cyrilies E. y Schamme (2021) El aprendizaje basado en Proyectos: Una capacitación docente vinculante. Páginas de Educación Scielo.edu.uy Buenos Aires.

Decreto Supremo (2020) 008 S.A. publicado en el diario Oficial Peruano.

Dubs R. (2021) La formación docente de una educación técnica competitiva Sapiens Revista universitaria de Investigación Revista upel.edu.ve Venezuela

Euler D. (2018) Herramientas de trabajo para el dialogo político y el diseño de proyectos de cooperación de desarrollo - partel comité de donantes para la formación profesional dual (D C $d V E T) \quad Z \quad$ Zúrich Suiza Zúrich

Fuentes R. (2020) Aplicación del programa curricular en el área de EPT sobre la orientación vocacional y satisfacción en la elección de la carrera profesional técnica universitaria del colegio experimental de aplicación de la $\begin{array}{llll}\text { Universidad Nacional de } & \text { Educación } & 2017\end{array}$ http://repositorio.une.edu.pe/handle/UNE/3326

García Cl. La inteligencia emocional en el desarrollo de la trayectoria académica del universitario. Rev. Cubana Edu. Superior [online]. 2020, vol.39, n.2 [citado 2021-09-27], e15. Disponible en: <http://scielo.sld.cu/scielo.php?script=sci_arttext\&pid=SO25743142020000200015\&lng=es\&nrm=iso>. Epub 01-Ago-2020. ISSN $0257-4314$

Heredia Y:(2020) El desarrollo emocional están importante como lo académico Instituto para el futuro de la educación tecnológica de Monterrey 
https://observatorio.tec.mx/edu-bits-blog/importancia-del-desarrolloemocional-estudiantes

Hernández R. Fernández C. y Baptista P. (2014). Metodología de la investigación. México: Mc Graw-Hill.

Hernández .B.; Gentile, A.; Herrera I. (2021) Participación juvenil para el desarrollo rural: análisis de un caso cubano en cooperativas agrarias. REVESCO. Revista de Estudios Cooperativos, vol. 137, e73864. https://dx.doi.org/10.5209/reve.73864.

Instituto Nacional de Estadística (2020). Informe anual 2020. Lima: INEI.

Lewis J. (2019) Ser adolescente en tiempo de Covid Riesgos en la salud mental Médico psiquiatra MP.36105 RNE 19255 escuela de psicología de la UCSP Cuantitativa-cualitativa y redacción de tesis. Colombia: DGP Editores

Melo L. y Silva C (2021) Centro de capacitación para jóvenes adolescentes revitalización urbana en nodo comercial de Chincha Alta http://repositorio.urp.edu.pe/handle/URP/4010 Universidad Ricardo Palma Lima Av. Benavides 5440 - Santiago de Surco Lima 33 Perú | Apartado postal $1801 \mid(0511) 7080000$

Ministerio de Educación (2021). Resolución Ministerial N 121-2021-MINEDU. Aprobar el documento normativo denominado disposiciones para la prestación del servicio en las instituciones y programas educativos públicos y privados de la educación básica de los ámbitos urbanos y rurales, en el marco de la emergencia sanitaria de la covid-19 Lima: Diario El Peruano.

Municipalidad de Mala (20199 Distrito de Mala de la provincia de Cañete región Lima

https://www.iperu.org/distrito-de-mala-provincia-de-canete

.OMS (2020) Vías de transmisión del virus de la COVID-19: repercusiones para las recomendaciones relativas a las precauciones en materia de prevención de 
las infecciones: Reseña científica. Ginebra. Retrieved from https://bit.ly/2MaAiHK

Pinto G. Plaza L. (2021) Determinar la necesidad de capacitación en el uso de tecnologías de la información y la comunicación para la formación docente- Universidad Espíritu docente escuelas localización 593 digital Publisher CEIT ISSN e 2588-0705 vol6 $N^{\circ} 1$

Revista Dialnet

Ryder G. (2020) Los jóvenes y la pandemia - director de la OIT https://www.ilo.org/global/about-theilo/newsroom/news/WCMS_753062/lang-- es/index.htm.

Sánchez J. Martín S. Bel P. y Lejarriaga G. (2018) Educación y formación en emprendimiento social: características y creación de valor social sostenible en proyectos de emprendimiento social. REVESCO. Revista de Estúdios Cooperativos,

Tercer Cuatrimestre, No 129, pp. 16-38. DOI: 10.5209/REVE.62492.

Sheposh R. (2019) Corona vírus (COVID-19) Salem Press Encyclopedia of Health.

Sorbara S. Baró S. Greco R. Preiti M. y Quinteros M. (2021). Doble presencia, entre la familia y el trabajo. Revista Científica Arbitrada de la Fundación Mente Clara, Vol. 6 (251).

DOI: $\underline{\text { https://doi.org/10.32351/rca.v6.251 }}$

Suárez G. (2020) ¿Las Familias o la familia desde el trabajo social? Teléfono de contacto: +58 4147334713 Ministerio del Poder Popular para la Educación Zona Educativa No 14 Mérida-Venezuela. Educere Artículos arbitrados ISSN 13164910 año $25 \mathrm{~N}^{\circ} 80$

Tamayo M. (2007). El proceso de la investigación científica. Buenos Aires: Limusa. 
Urzúa A., Vera P., Caqueo A. y Polanco R. (2020). La psicología en la prevención y manejo del COVID-19. Aportes desde la evidencia inicial. Terapia Psicológica, 38(1), 103-118.

Velasco G. (2020) Dirección estratégica a través del emprendimiento y modelo de negocio Revista iberoamericana de contaduría económica y administración ISSN 2007- 9507 artículo científico htt://doi.org/90.90.23913/riceav9i17 145 Universidad Autónoma de ciudad Juárez México.

Vicentini I. (2020). La educación superior en tiempos de COVID-19: Aportes de la Segunda Reunión del Diálogo Virtual con Rectores de Universidades Líderes de América Latina 